\title{
Avaliação da segurança do extrato hidroalcoólico de partes aéreas de Plectranthus neochilus Schlechter, Lamiaceae e entrecascas de Cnidoscolus quercifolius Pohl, Eupharbiaceae em roedores
}

\author{
[Evaluation of the safety of the hydroalcoholic extract of aerial parts of Plectranthus neochilus \\ Schlechter, Lamiaceae and Cnidoscolus quercifolius Pohl, Eupharbiaceae in rodents]
}

\section{"Artigo Científico/Scientific Article"}

\author{
Michelle Suassuna de Azevedo Rêgo ${ }^{1 *}$, Vanessa Carla Lima da Silva² ${ }^{2}$ Carina Scanoni Maia ${ }^{3}$, \\ Mirian Nogueira Teixeira ${ }^{1}$, Melânia Loureiro Marinho ${ }^{4}$, Evilda Rodrigues de Lima ${ }^{1}$
}

\begin{abstract}
${ }^{1}$ Departamento de Medicina Veterinária, Universidade Federal Rural de Pernambuco, Recife-PE, Brasil. ${ }^{2}$ Departamento de Medicina Veterinária, Centro Universitário Brasileiro (UNIBRA), Recife-PE, Brasil. ${ }^{3}$ Departamento de Histologia e Embriologia, Centro de Ciências Biológicas, Universidade Federal de Pernambuco, Recife-PE, Brasil.

${ }^{4}$ Centro de Saúde e Tecnologia Rural, Universidade Federal de Campina Grande, Patos-PB, Brasil.

*Autor para correspondência/Corresponding author: E-mail: michellesuassuna@ hotmail.com
\end{abstract}

\section{Resumo}

Objetivou-se avaliar a toxicidade aguda do extrato hidroalcoólico de Plectranthus neochilus (boldo-gambá) e Cnidoscolus quercifolius (favela) e a toxicidade dérmica de uma Formulação em Gel (FG) produzida a partir dos extratos. Na toxicidade aguda utilizou-se nove camundongos Swiss, fêmeas, em três grupos: G1 - com extrato de $P$. neochilus e $C$. quercifolius; G2 - C. quercifolius e G3 - P. neochilus, uma vez via gavagem $(2 \mathrm{~g} / \mathrm{kg})$. Na toxicidade dérmica utilizou-se 35 ratos Wistar, em cinco grupos, nos quais foram induzidos uma lesão dérmica e expostos a FG da associação de P. neochilus e C. quercifolius (FGBF) 2,5\%, 5\% ou 10\%. Os grupos controles receberam gel fitoterápico ou veículo carbopol. Foram observados durante 14 dias. Após a administração aguda dos extratos não houve mortalidade nem sinais de intoxicação. A aplicação tópica em pele lesionada não induziu irritação local. Mas, foram observadas alterações significativas no perfil hematológico e bioquímico nos tratados com a FGBF quando comparados ao controle negativo (carbopol). A contagem das plaquetas nos tratados com as FGBF 2,5\% ou 10\%, apresentaram um aumento de $13,22 \%$ e 22,94\%, respectivamente; ALT (95,39\%) e AST (38,55\%) na FGBF 5\% e FA na FGBF 2,5\% (46,24\%) e 5\% $(31,80 \%)$ e diminuição do colesterol nas FGBF 2,5\% (40,02\%), $5 \%$ (38,59\%) e 10\% (34,06\%). Os extratos de $P$. neochilus e $C$. quercifolius quando administrado via gavagem causou toxicidade aguda relativamente baixa revelando uma $\mathrm{DL}_{50}$ superior $2 \mathrm{~g} / \mathrm{kg}$. Os ratos tratados com o gel apresentaram alterações hematológicas e bioquímicas mas dentro do padrão de normalidade para a espécie.

Palavras-chave: fitoterapia; toxicidade; plantas medicinais.

\begin{abstract}
The objective was to evaluate the acute toxicity of the hydroalcoholic extract of Plectranthus neochilus (boldopossum) and Cnidoscolus quercifolius (favela) and the dermal toxicity of a Gel Formulation (GF) produced from the extracts. In acute toxicity nine female Swiss mice were used in three groups: G1 - with extract of P. neochilus and C. quercifolius; G2 - C. quercifolius and G3 - P. neochilus, once via gavage $(2 \mathrm{~g} / \mathrm{kg})$. In the dermal toxicity 35 Wistar rats were used in five groups, in which a dermal lesion was induced and exposed to FG of the association of $2.5 \%, 5 \%$, or $10 \%$ of $P$. neochilus and C. quercifolius (FGBF). Control groups received herbal gel or carbopol vehicle. They were observed for 14 days. After acute administration of the extracts there was no mortality or signs of intoxication. Topical application to injured skin did not induce local irritation. However, significant alterations were observed in the hematological and biochemical profile in the treated with FGBF when compared to the negative control (carbopol). The platelet count in the treated patients
\end{abstract}


with $2.5 \%$ or $10 \%$ FGBF showed an increase of $13.22 \%$ and $22.94 \%$, respectively; ALT $(95.39 \%)$ and AST (38.55\%) in FGBF 5\% and FA in FGBF 2.5\% (46.24\%) and 5\% (31.80\%) and decrease in cholesterol in FGBF 2, 5\% (40.02\%), 5\% (38.59\%) and 10\% (34.06\%). Extracts of P. neochilus and C. quercifolius when administered via gavage caused relatively low acute toxicity revealing an LD $50 \mathrm{of} 2 \mathrm{~g} / \mathrm{kg}$. The rats treated with the gel presented hematological and biochemical alterations but within the standard of normality for the species.

Keywords: phytotherapy; toxicity; medicinal plants.

\section{Introdução}

As plantas medicinais têm finalidades terapêuticas e/ou profiláticas importantes para várias doenças, sendo uma das formas de tratamento mais antigas. O seu uso vem sendo propagado do início da civilização até os dias de hoje (Rodrigues et al., 2010). No entanto, nem todas as propriedades terapêuticas são conhecidas e podem causar intoxicação em seres humanos e animais, a depender da dose e forma de administração (Martins et al., 2012).

A espécie Plectranthus neochilus Schlechter, conhecida por boldo-gambá, pertence ao gênero Plectranthus, família Lamiaceae (subfamília Nepetoideae), contendo cerca de 350 espécies de ervas e arbustos perenes nativas de regiões tropicais da África, Ásia e Austrália (Codd, 1985). Várias espécies do gênero Plectranthus são cultivadas como tubérculos comestíveis, condimentos, fragrâncias para xampus, plantas ornamentais ou como fontes de óleos essenciais (Ascensão et al., 1999), preparações farmacêuticas, perfumaria e cosméticos (Ascensão et al., 1998), componentes de preparações cosméticas patenteadas como anti-sinais (Okamoto et al., 2005), e produtos para a estimulação do crescimento capilar (Kaneda et al., 2005). As diversas utilizações na medicina tradicional incluem tratamentos para cefaléia, feridas, queimaduras, dermatite, alergias, picadas de inseto e de escorpião e como agente anti-séptico (Ascensão et al., 1998, 1999).

Cnidoscolus quercifolius Pohl é popularmente conhecida como favela ou faveleira, pertencente ao gênero Plectranthus, família Euphorbiaceae, está presente nos estados do Piauí, Ceará, Rio Grande do Norte, Paraíba, Pernambuco, Bahia e dentre outros (Duque, 2004). O gênero Cnidoscolus compreende cerca de 50-75 espécies, distribuídas excepcionalmente na América tropical e concentradas, principalmente, no México e Nordeste do Brasil (Webster e Rupert, 1973). Essas espécies possuem diversas propriedades medicinais, dentre elas, atividade antitumoral, anti-

inflamatória para o sistema genito-urinário, antisséptica e no tratamento de infecções renais, dermatológicas e lesões oftalmológicas, contusões, fraturas, feridas, verrugas, disenteria, hemorragia, apendicite e reumatismo (Agra et al., 2008). Na medicina popular do Nordeste, a decocção, a infusão e maceração das cascas e entrecascas do caule são empregadas contra inflamações ovarianas e inflamações gerais (Lorenzi e Matos, 2002).

Toxicidade oral aguda é o método utilizado para avaliar a toxicidade produzida por uma substância teste quando administrada durante um período não superior a 24 horas, seguido da observação dos animais por 14 dias após essa administração (OECD, 2001). Estes testes demonstraram informações adequadas de toxicidade aguda e letalidade utilizando um número menor de animais comparado ao teste clássico de DL 50 . Embora o principal ainda seja a letalidade, estes testes diminuem o número de animais e o sofrimento e em alguns casos nos dizem informações adequadas para a classificação toxicológica das substâncias estudadas (Dipasquale e Hayes, 2001). Diante da importância da P.neochilus e da C. quercifolius como plantas medicinais, objetivou-se obter extratos e analisar a toxicidade aguda e dérmica destas espécies vegetais em roedores.

\section{Material e Métodos}

As partes aéreas de Plectranthus neochilus e entrecascas de Cnidoscolus quercifolius foram coletadas de espécimes localizados na mesorregião do Sertão Paraibano, município de Patos - Brasil

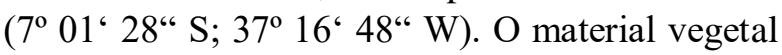
coletado foi identificado pelo Departamento de Engenharia Florestal da Universidade Federal Rural de Pernambuco (UFRPE) e uma exsicata de cada depositada no Herbário da UFRPE sob os números 21717 e 21101, respectivamente.

Para a obtenção do extrato hidroalcoólico foram utilizadas $500 \mathrm{~g}$ das partes aéreas de $P$. 
neochilus e $500 \mathrm{~g}$ da entrecasca de C. quercifolius, desidratadas em estufa de circulação de ar a $60^{\circ} \mathrm{C}$, trituradas, maceradas e pesadas. Em seguida foi adicionado 1L (EtOH: ÁGUA 1:1), deixando em repouso por 72 horas à temperatura ambiente, para posterior filtração do material, concentração em rotoevaporador, congelamento e liofilização para obtenção dos extratos hidroalcoólicos (EH) de $P$. neochilus e $C$. quercifolius que foram armazenados a $-20^{\circ} \mathrm{C}$. Procedeu-se ainda uma triagem fitoquímica nos extratos.

Para a obtenção do veículo foi utilizado carbopol a $1 \%$. O gel apresentou propriedades características como boa viscosidade e o $\mathrm{pH}$ foi ajustado para 6,0 . Para cada $100 \mathrm{mg}$ do veículo foi utilizado $2,5 \mathrm{mg}$ do extrato hidroalcoólico de $P$. neochilus associada a $2,5 \mathrm{mg}$ do extrato hidroalcoólico de C. quercifolius (FGBF 2,5\%); $5 \mathrm{mg}$ de cada extrato (FGBF 5\%), e 10mg de cada extrato (FGBF 10\%).

No modelo experimental da toxicidade oral aguda foram utilizados nove camundongos Swiss (Mus musculus albinus) adultos jovens, fêmeas, peso entre 19 e $25 \mathrm{~g}$, provenientes do Biotério Central da Universidade Federal Rural de Pernambuco. Foram agrupados em três grupos aleatórios ( $\mathrm{n}=3$ /grupo): Grupo1 - animais tratados com boldo-gambá e favela juntos; Grupo 2 animais tratados com favela e Grupo 3 - animais tratados com boldo-gambá. Os animais foram mantidos na Sala de Experimentação do Laboratório de Farmacologia de Produtos Bioativos do Centro de Ciências Biológicas da UFPE, mantidos em gaiolas de propileno translúcido $(40 \times 32 \times 16 \mathrm{~cm})$, com tampa de arame zincado e cama de maravalha, sob condições controladas de iluminação (ciclo $12 \mathrm{~h}$ claro/escuro), temperatura $\left(22 \pm 2^{\circ} \mathrm{C}\right)$ e umidade $(76 \pm 5,4 \%)$, água e dieta (Presence ${ }^{\circledR}$ ) ad libitum. Para o tratamento com administração via gavagem com utilização de sondagem orogástrica, utilizou-se volume máximo de administração de $1 \mathrm{~mL} / 100 \mathrm{~g}$ de peso corporal.

$\mathrm{O}$ teste de toxicidade oral aguda foi realizado segundo metodologia descrita no Guia 423 das diretrizes da OECD. Em todos os casos foi administrada uma única dose $(2 \mathrm{~g} / \mathrm{kg}$ de cada extrato, por gavagem). Os animais, os quais estavam em jejum durante 8 horas, foram pesados antes dos tratamentos, no $4^{\circ}, 7^{\circ}, 10^{\circ}, 13^{\circ}$ e $15^{\circ}$ dia de estudo.

Os animais foram observados individualmente, após a primeira dose, por cerca de 30 minutos, e periodicamente durante as primeiras 24h, com atenção especial durante as primeiras 4 horas, e diariamente depois disso, num total de 14 dias. Os parâmetros analisados foram: avaliação clínica (modificações na pele, pelos olhos, mucosas, alterações respiratórias, circulatórias e dos sistemas nervoso central e periférico) realizada diariamente; avaliação de efeitos gerais, ou seja, alterações no estado de consciência e disposição, no sistema motor, o tônus muscular, no sistema nervoso central (tremores, convulsão e sedação) e no sistema nervoso autônomo (lacrimejamento, salivação, ptose, piloereção e respiração).

No décimo quinto dia, os animais foram eutanasiados por aprofundamento anestésico (cetamina $60 \mathrm{mg} / \mathrm{kg}$ e xilazina $10 \mathrm{mg} / \mathrm{kg}$ ) e posteriormente exsanguinação por punção venosa cardíaca, submetidos à necropsia, realizando-se análise macroscópica e pesagem em balança analítica de precisão, do coração, baço, rins e fígado, buscando-se possíveis sinais de alterações sugestivas de toxicidade. Após esse procedimento, foi realizada a classificação do risco toxicológico com base na OECD 423 (2001). de acordo com a tabela do Globally Harmonised Classification System (GHS).

No modelo experimental da toxicidade dérmica em pele lesionada foram utilizados 35 ratos Wistar (Rattus norvegicus albinus) machos, peso corpóreo de 220 a 250g, provenientes do biotério central da UFPE. Todos os animais da pesquisa foram mantidos em ambiente climatizado $\left(20-22^{\circ} \mathrm{C}\right)$, sob iluminação artificial, com ciclo claro-escuro a cada 12 horas, acondicionados em gaiolas metabólicas individuais, com livre acesso a alimentação e água. Os primeiros 15 dias antes da cirurgia foram considerados como período de aclimatação, visando estabilizar os animais na sala de experimentação.

Para a cirurgia (jejum prévio de 12 horas), os ratos Wistar $(\mathrm{n}=35)$ foram pesados, em seguida, anestesiados com cetamina $(10 \mathrm{mg} / \mathrm{kg})$ e xilazina $(3 \mathrm{mg} / \mathrm{kg})$ (Andrade, 2002), por via intraperitoneal, colocados em decúbito ventral e submetidos a tricotomia manual e antissepsia com $0,1 \%$ de álcool iodado na linha média dorsal da região cervical. A área da incisão foi marcada com uma caneta de marcação e molde metalálico vazado $\left( \pm 78,5 \mathrm{~mm}^{2}\right)$. Para remover a pele, tecido subcutâneo, fáscia e panículo carnoso, utilizou-se tesoura reta de Iris e pinça de Adson. Depois da incisão, um anel de contenção feito de material atóxico e hipoalergênico de silicone foi suturado ao 
redor da ferida cirúrgica usando de 4 a 6 pontos isolados simples com fio monofilamentoso de nylon 4.0 agulhado, distribuídos simetricamente (Galiano et al., 2004, adaptado por Franco, 2012), de modo que a ferida permanecesse no centro.

Após a cirurgia, os ratos foram distribuídos aleatoriamente em cinco grupos ( $n=7 /$ grupo): Grupo 1, controle positivo, onde utilizou-se um gel fitoterápico já existente no mercado $(10 \mathrm{~mL}$ de tintura de Calendula officinalis $\mathrm{L}$.; $10 \mathrm{~mL}$ de tintura de Stryphnodendron barbatiman Mart.; $5 \mathrm{~mL}$ de tintura de Symphyntum officinalis L.; $5 \mathrm{~mL}$ de tintura de Aloe vera; $3 \mathrm{~mL}$ de tintura de Matricaria chamomilla L.; $5 \mathrm{~mL}$ de tintura de Echinacea angustifólia DC.; $8 \mathrm{~mL}$ de tintura de própolis, $100 \mathrm{~g}$ do gel carbopol; Grupo 2, controle negativo, utilizou apenas o veículo carbopol; os Grupos 3, 4 e 5 formaram grupos da associação específica com a incorporação do extrato $P$. neochilus, e $C$. quercifolius a 2,5\%, 5\% e $10 \%$ ao gel de carbopol.

No final do experimento, os animais foram submetidos a jejum prévio de 8 horas, sedados com cetamina $(10 \mathrm{mg} / \mathrm{kg})$ e xilazina $(3 \mathrm{mg} / \mathrm{kg})$ e submetidos a eutanásia por decapitação, procedendo-se a coleta para realização de exames hematológicos, bioquímicos e histopatológios.

Foram coletados $4,0 \mathrm{~mL}$ do sangue dos animais em dois tubos diferentes: um com anticoagulante EDTA (Doles Reag. Equip. para Laboratórios Ltda, Goiânia, Brasil) com 1,0mL para a realização de hemograma e $3,0 \mathrm{~mL}$ em um tubo seco para exames bioquímicos. Para a obtenção do soro, a amostra foi imediatamente centrifugada a 7500 r.p.m. por 15 minutos e congelada a $-20^{\circ} \mathrm{C}$ por até 72 horas. Os parâmetros hematológicos (hemograma e contagem de plaquetas) foram determinados imediatamente após a coleta através do analisador automático de celúlas hematologicas ABX Micros 60 (HORIBA ABX Diagnostics, Montpellier, França).

Os parâmetros bioquimicos gamaglutamiltranspeptidase (GGT), Alanina aminotransferase (ALT), Aspartato aminotransferase (AST), fosfatase alcalina (FA), uréia, creatinina, ácido úrico, colesterol, triglicérides, glicose, proteínas totais e albumina, foram determinados com o auxílio do analisador automático Labmax 240 (Tokyo Boeki Machinery Ltd, Medical Project Department - Tokyo, Japão) e kits comerciais da marca Labtest ${ }^{\circledR}$.

Os animais foram necropsiados, e removidos rins, baço, coração e fígado para avaliação macroscópica, pesagem e análise histopatológica.
Os resultados numéricos foram expressos por média \pm erro padrão da média e para fins estatísticos $(\mathrm{p}<0,05)$. A normalidade das medidas foi avaliada através do teste de Kolmogorov Smirnov. Os dados paramétricos foram submetidos à Análise de Variância (ANOVA) duas vias, com aplicação do pós-teste de Bonferroni. Os dados foram analisados estatisticamente pelo programa Prism ${ }^{\circledR} 4.00$ (GraphPad Software Inc., San Diego, California, US).

\section{Resultados e Discussão}

O extrato hidroalcoólico de $P$. neochilus apresentou-se como um resíduo claro e viscoso com rendimento de $26,6 \%$ e o extrato hidroalcólico de $C$. quercifolius, como um resíduo escuro e viscoso com rendimento de $10 \%$. A triagem fitoquímica confirmou a presença de flavanóides em ambos os extratos. Não foi observado morte ou qualquer outro sinal de toxicidade nos animais tratados com o extrato de $P$. neochilus e entrecascas de $C$. quercifolius na dose de $2 \mathrm{~g} / \mathrm{kg}$ administrados por gavagem.

Gomes et al. (2014) avaliando a toxicidade aguda do extrato etanólico das cascas de $C$. quercifolius, não observaram mortes dos camundongos que receberam a dose $5 \mathrm{~g} / \mathrm{kg}$ por via oral, indicando baixa toxicidade dos extratos estudados. Nas observações clínicas realizadas durante o experimento como alterações dos sistemas respiratório, nervoso central e periférico somático e autônomo e locomotor, não foram observadas alterações nesses sistemas e nem alterações comportamentais nos animais avaliados.

As observações descritas na triagem comportamental servem para facilitar a investigação da possível atividade do extrato no sistema nervoso central, bem como indicar os efeitos do mesmo no comportamento dos animais (Almeida, 2006). Na avaliação comportamental de camundongos que receberam o extrato da casca de favela na dose de $5 \mathrm{~g} / \mathrm{kg}$ por via oral, os animais (fêmeas e machos) demonstraram as seguintes alterações: ambulação diminuída, ptose palpebral e diarreia. Os camundongos (machos e fêmeas) que receberam a dose $5 \mathrm{~g} / \mathrm{kg}$ do extrato das folhas de favela, durante a a avaliação comportamental não demonstraram alterações no comportamento (Gomes et. al., 2014).

$\mathrm{Na}$ Figura 1 observou-se a evolução ponderal do peso corporal (g) de camundongos fêmeas durante o estudo da toxicidade aguda. A 
administração do extrato de $P$. neochilus, e $C$. quercifolius (associados e/ou isolados) na dose de $2 \mathrm{~g} / \mathrm{kg}$ não gerou diferenças estatisticamente significativas entre os grupos estudados ( $p>0,05) \mathrm{em}$ relação ao ganho de peso corporal dos camundongos. Sangeetha et al. (2013) observaram a perda de peso corporal como sendo um sinal característico observado na maioria dos animais expostos a substâncias tóxicas, essa diminuição do peso geralmente se manifesta dentro de alguns dias após a exposição, e resulta numa redução substancial do tecido adiposo e do tecido muscular.

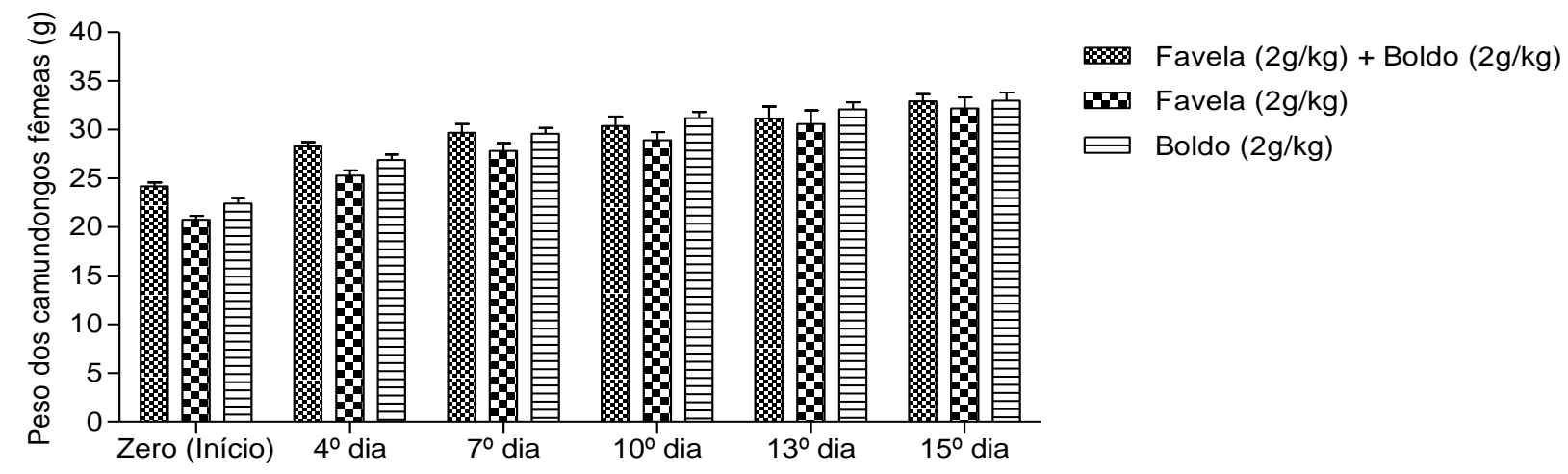

Figura 1. Evolução ponderal do peso corporal (g) de camundongos fêmeas durante o estudo da toxicidade oral aguda dos extratos hidroalcoólicos de Plectranthus neochilus (boldo-gambá) e/ou Cnidoscolus quercifolius (favela).

Em estudo Gomes et al. (2014) avaliaram o peso corporal de camundongos que ingeriram o extrato hidroalcoólico da casca da favela durante 14 dias e foram detectadas diferenças estatísticas nos grupos de fêmeas na dose de $5 \mathrm{~g} / \mathrm{kg}$ por via oral. Já os tratados com o extrato das folhas de favela apresentaram diferenças estatísticas no grupo $5 \mathrm{~g} / \mathrm{kg}$ por via oral em machos. Segundo Jahn e Geunzel (1997) o acompanhamento da massa corporal do animal é um importante indicador para a avaliação da toxicidade de substâncias exógenas.

Os pesos relativos dos órgãos dos camundongos tratados com os extratos hidroalcoólicos de Plectranthus neochilus (boldogambá) e/ou Cnidoscolus quercifolius (favela) estão representados na Figura 2. Não houve diferenças estatísticas em relação ao peso do coração, rins e baço, entretanto, verificaram-se aumento estatisticamente significativo no peso do fígado dos animais tratados apenas com o extrato de Plectranthus neochilus (boldo-gambá). Na análise macroscópica não foi visualizada alterações da consistência do fígado. Gomes et al. (2014) analisando os pesos dos órgãos de camundongos machos e fêmeas tratados com Cnidoscolus quercifolius na dose de $5 \mathrm{~g} / \mathrm{kg}$ por via oral, não observaram nenhuma alteração macroscópica nos órgãos avaliados, demonstrando assim, que não houve toxicidade.

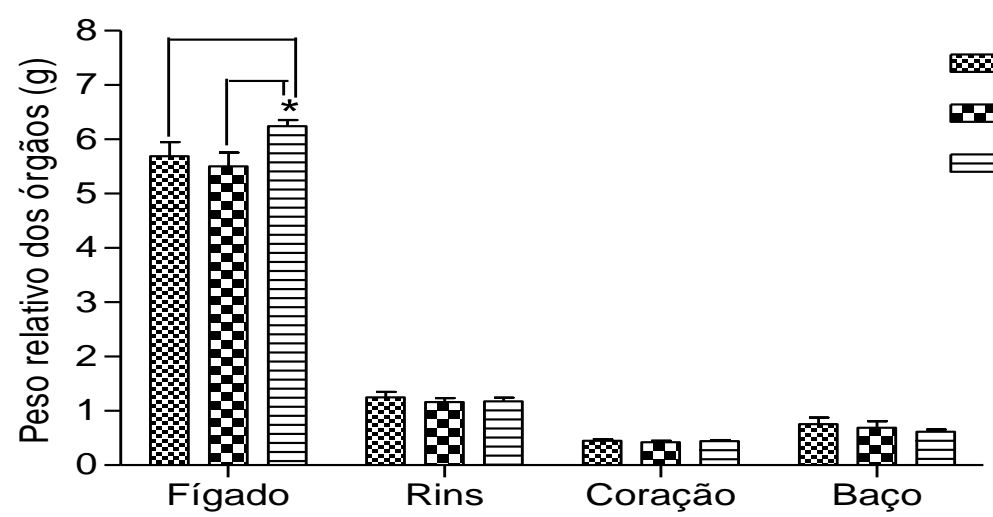

Figura 2. Peso relativo dos órgãos $(\mathrm{g} / 100 \mathrm{~g})$ de camundongos fêmeas durante o estudo de toxicidade oral aguda com extratos hidroalcoólicos de Plectrantus neochilus (boldo-gambá) e/ou Cnidoscolus quercifolius (favela). 
As avaliações clínicas diárias mostraram ausência de toxicidade dérmica com adequada recuperação, com manutenção do estado geral, presença de atividade física, disposição para alimentar-se e ausência de mortes nos animais tratados com FGBF a $2,5 \%, 5 \%$ ou $10 \%$ e grupos controle positivo e negativo.

Na Figura 3 observa-se a evolução do peso corporal dos ratos Wistar machos durante o estudo, sendo estatisticamente significativo no grupo da FGBF a $10 \%$ nas pesagens zero, sete, dez e treze dias comparados aos demais grupos. No entanto, estes resultados podem estar relacionados ao menor peso corpóreo dos ratos desde o dia zero comparados com os demais grupos e por não apresentarem perda de peso. Possivelmente, devido ao mesmo manejo a que foram submetidos, não afetando a ingestão de água ou alimentos, bem como reações clínicas significativas, como: prurido intenso, edema, eritema, diarreia, vômitos, mudanças de comportamento ou mortes. Animais que apresentam lesões dermatológicas, acompanhadas de prurido e dor, não se alimentam bem e apresentam perda de peso (Brito, 1995). Este fato não foi observado nos animais que foram submetidos aos tratamentos durante os 14 dias.

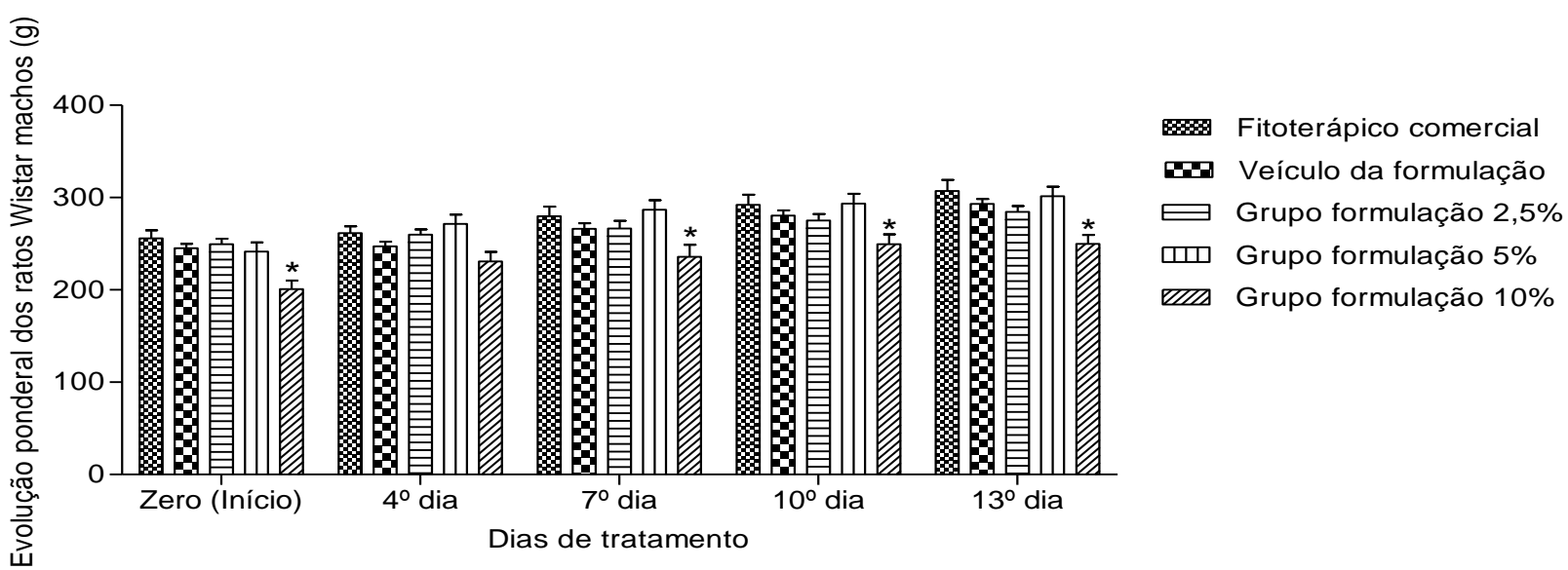

Figura 3. Evolução ponderal do peso corporal (g) de ratos Wistar machos durante o estudo da toxicidade dérmica dos grupos controle positivo (fitoterápico comercial), controle negativo (veículo), Formulação em Gel da associação de Plectranthus neochilus (boldo-gambá) e/ou Cnidoscolus quercifolius (favela) nas concentrações 2,5\%, 5\% ou 10\% .

A Figura 4 apresenta o peso dos órgãos (fígado, rins, coração e baço) dos ratos no final do experimento, verificando resultados estatisticamente significativos para o fígado dos animais da FGBF 5\% e 10\%. Klaassen e Watkins
(2001) relataram que a toxicidade subcrônica estabelece a existência ou não de efeitos adversos e posteriormente identifica e caracteriza os órgãos afetados determinados pelo efeito cumulativo da substância administrada.

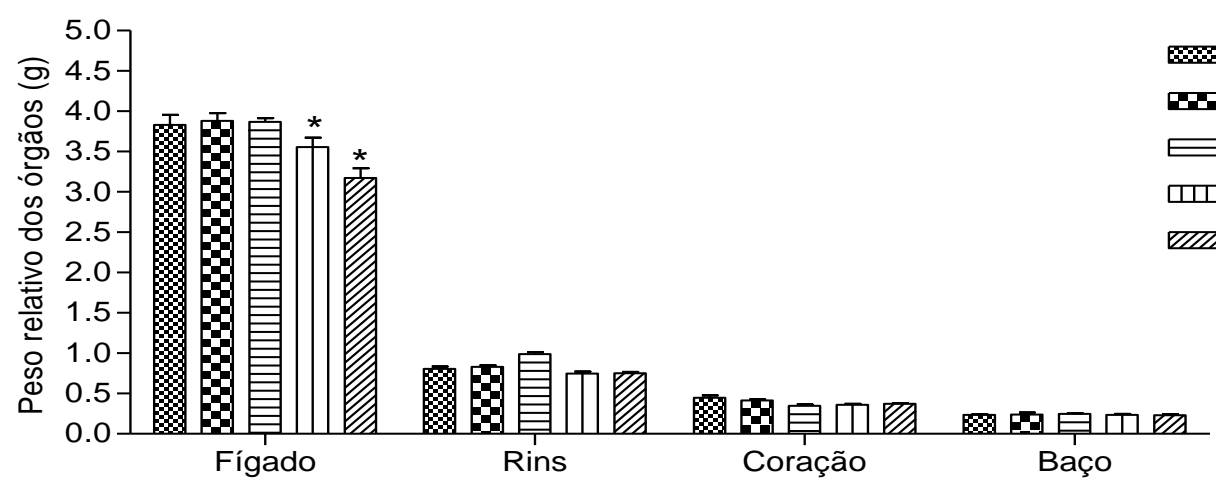

Figura 4. Peso relativo dos órgãos $(\mathrm{g} / 100 \mathrm{~g})$ dos ratos Wistar machos durante o estudo de toxicidade dérmica dos grupos controle positivo (fitoterápico comercial), controle negativo (veículo), Formulação em Gel da associação de Plectranthus neochilus (boldo-gambá) e/ou Cnidoscolus quercifolius (favela) nas concentrações 2,5\%, 5\% ou 10\%.

Analisando a Tabela 1 observa-se que houve diferença estatística quanto a variável contagem de plaqueta nos ratos dos Grupo FGBF 2,5\% e no Grupo FGBF $10 \%$. As demais variáveis 
relacionadas com o hemograma em todos os grupos não apresentaram diferenças estatísticas, demonstrando assim que as formulações não tiveram influência sobre esses parâmetros (Werner e Grose, 2003; Eming et al., 2007).

Tabela 1. Parâmetros hematológicos de ratos Wistar machos tratados com o gel fitoterápico comercial, veículo da formulação, ou $\operatorname{FGBF}(2,5 \%, 5 \%$ ou $10 \%)$.

\begin{tabular}{|c|c|c|c|c|c|c|}
\hline \multirow[b]{2}{*}{ Parâmetros } & \multirow{2}{*}{$\begin{array}{c}\text { Valores do } \\
\text { Biotério do } \\
\text { LTF- } \\
\text { UFPB }^{\#} \\
\end{array}$} & \multicolumn{5}{|c|}{ Grupos } \\
\hline & & $\begin{array}{c}\text { Fitoterápico } \\
\text { comercial }\end{array}$ & $\begin{array}{l}\text { Veículo da } \\
\text { formulação }\end{array}$ & FGBF 2,5\% & FGBF 5\% & FGBF 10\% \\
\hline $\begin{array}{l}\text { Hemácias } \\
\left(10^{6} / \mathrm{mm}^{3}\right)\end{array}$ & $7,3 \pm 0,1$ & $7,87 \pm 0,10$ & $7,21 \pm 0,28$ & $7,90 \pm 0,21$ & $7,41 \pm 0,12$ & $7,53 \pm 0,15$ \\
\hline Hemoglobina (g/dL) & $15,0 \pm 0,2$ & $13,66 \pm 0,12$ & $12,41 \pm 0,34$ & $12,39 \pm 0,27$ & $12,31 \pm 0,24$ & $13,11 \pm 0,14$ \\
\hline $\begin{array}{l}\mathrm{VCM} \\
\left(\mu \mathrm{m}^{3}\right)\end{array}$ & $48,0 \pm 0,3$ & $55,71 \pm 0,42$ & $55,14 \pm 0,40$ & $55,86 \pm 0,40$ & $56,43 \pm 0,43$ & $56,00 \pm 0,79$ \\
\hline $\begin{array}{l}\mathrm{HCM} \\
(\mathrm{pg})\end{array}$ & $20,0 \pm 0,2$ & $17,36 \pm 0,15$ & $17,29 \pm 0,24$ & $15,66 \pm 0,18$ & $16,71 \pm 0,11$ & $17,44 \pm 0,29$ \\
\hline $\begin{array}{l}\text { CHCM } \\
\text { (g/dL) }\end{array}$ & $42,0 \pm 0,2$ & $31,16 \pm 0,27$ & $31,26 \pm 0,30$ & $27,96 \pm 0,19$ & $29,40 \pm 0,18$ & $31,13 \pm 0,18$ \\
\hline $\begin{array}{l}\text { RDW } \\
(\%)\end{array}$ & $13,0 \pm 0,2$ & $12,31 \pm 0,10$ & $12,21 \pm 0,09$ & $12,91 \pm 0,13$ & $12,89 \pm 0,26$ & $12,87 \pm 0,60$ \\
\hline $\begin{array}{l}\text { Plaquetas } \\
\left(\mathrm{mm}^{3}\right)\end{array}$ & $730 \pm 33,0$ & $616,14 \pm 17,98$ & $598,29 \pm 33,13$ & $677,43 \pm 25,10^{*}$ & $639,86 \pm 14,47$ & $735,57 \pm 41,07 *$ \\
\hline $\begin{array}{l}\text { Leucócitos } \\
\left(\mathrm{mm}^{3}\right)\end{array}$ & $6,3 \pm 0,5$ & $7,60 \pm 0,46$ & $7,90 \pm 0,82$ & $7,09 \pm 0,44$ & $4,84 \pm 0,27$ & $3,81 \pm 0,25$ \\
\hline $\begin{array}{l}\text { Monócitos } \\
(\%)\end{array}$ & $5,1 \pm 0,3$ & $11,3 \pm 0,11$ & $8,4 \pm 0,06$ & $11,1 \pm 0,10$ & $12,6 \pm 0,07$ & $14,9 \pm 0,05$ \\
\hline $\begin{array}{l}\text { Granulócitos } \\
(\%)\end{array}$ & $27,0 \pm 1,5$ & $29,1 \pm 0,31$ & $31,7 \pm 0,41$ & $28,8 \pm 0,14$ & $18,0 \pm 0,06$ & $16,8 \pm 0,09$ \\
\hline $\begin{array}{l}\text { Linfócitos } \\
(\%)\end{array}$ & $67,0 \pm 1,6$ & $59,6 \pm 0,31$ & $59,9 \pm 0,45$ & $60,0 \pm 0,35$ & $69,4 \pm 0,21$ & $68,3 \pm 0,16$ \\
\hline
\end{tabular}

Após a ocorrência da lesão, inicia-se o extravasamento sanguíneo que preenche a área lesada com plasma e elementos celulares, principalmente plaquetas. Agregação plaquetária e a coagulação sanguínea geram um tampão, rico em fibrina que além de restabelecer a homeostasia e formar uma barreira contra invasão de microorganismos, organiza matriz provisória necessária para a migração celular. Essa matriz servirá como reservatório de citocinas e fatores de crescimento que serão liberados durante as fases seguintes do processo cicatricial (Werner e Grose, 2003; Eming et al., 2007).

$\mathrm{Na}$ avaliação da toxicidade de plantas medicinais é de fundamental importância avaliar as alterações hematológicas, visto que essas permitem a detecção de alterações fisiopatológicas de animais em diferentes condições de estresse e são capazes de indicar a toxicidade induzida por hemólise (Nussey et al., 1995).

Estudos afirmaram que os valores dos parâmetros hematológicos (eritrócitos, hemoglobina, concentração de hemoglobina corpuscular média (CHCM), basófilos, eosinófilos, leucócitos, linfócitos, monócitos e neutrófilos), após a administração do extrato da casca da favela na dose de $5 \mathrm{~g} / \mathrm{kg}$ por via oral em fêmeas, causou alterações na contagem dos neutrófilos e houve diferença estatística (Gomes et al., 2014).

Dentre os efeitos adversos envolvidos no uso de plantas medicinais, podemos citar as alterações hematológicas, onde algumas plantas podem desencadear reações de toxicidade sanguínea, detectáveis através de exames laboratoriais, sendo possível avaliar os efeitos tóxicos destes elementos no sangue (Ferreira et al., 2013).

Analisando a Tabela 2 verificou-se que houve diferenças estatisticamente significativas nas variáveis colesterol nos ratos dos Grupos FGBF $2,5 \%, 5 \%$ e $10 \%$ com diminuição dos valores comparados aos da literatura e aumento da FA na FGBF $2,5 \%$ e $5 \%$. A FA é encontrada em praticamente todos os tecidos do corpo, mas que se apresenta, sobretudo no fígado, nas vias biliares e nos ossos (Meyer e Harvey, 1998). Essas variáveis podem ter sido influenciadas pela ação do Boldo- 
gambá que é indicado para insuficiência hepática e dispepsia e a Favela em ter ação colagoga, colerética e hepática. Essas plantas são recomen- dadas para diminuição dos níveis de colesterol e seu consumo excessivo podem provocar efeitos tóxicos (Lorenzi e Matos, 2002).

Tabela 2. Parâmetros bioquímicos de ratos Wistar machos tratados com o gel fitoterápico comercial, veículo da formulação, ou FGBF $(2,5 \%, 5 \%$ ou $10 \%)$.

\begin{tabular}{|c|c|c|c|c|c|c|}
\hline \multirow[b]{2}{*}{ Parâmetros } & \multirow{2}{*}{$\begin{array}{l}\text { Valores do } \\
\text { Biotério do } \\
\text { LTF-UFPB }\end{array}$} & \multicolumn{5}{|c|}{ Grupos } \\
\hline & & $\begin{array}{c}\text { Fitoterápico } \\
\text { comercial }\end{array}$ & $\begin{array}{l}\text { Veículo da } \\
\text { formulação }\end{array}$ & FGBF 2,5\% & FGBF $5 \%$ & $\begin{array}{c}\text { FGBF } \\
10 \%\end{array}$ \\
\hline $\begin{array}{l}\text { Glicose } \\
(\mathrm{mg} / \mathrm{dL})\end{array}$ & $117,4 \pm 2,8$ & $128,67 \pm 5,08$ & $\begin{array}{c}136,49 \pm 5,4 \\
3\end{array}$ & $148,60 \pm 5,01$ & $141,91 \pm 1,20$ & $\begin{array}{c}127,88 \pm 3,9 \\
9\end{array}$ \\
\hline $\begin{array}{l}\text { Colesterol } \\
(\mathrm{mg} / \mathrm{dL})\end{array}$ & $62,2 \pm 2,3$ & $84,04 \pm 4,07$ & $76,05 \pm 0,68$ & $45,61 \pm 1,62 *$ & $46,70 \pm 2,03 *$ & $\begin{array}{c}50,14 \pm 2,90 \\
*\end{array}$ \\
\hline $\begin{array}{l}\text { Triglicérides } \\
(\mathrm{mg} / \mathrm{dL})\end{array}$ & $96,0 \pm 5,5$ & $90,78 \pm 14,11$ & $80,68 \pm 8,85$ & $99,52 \pm 9,44$ & $75,14 \pm 17,56$ & $72,98 \pm 6,58$ \\
\hline $\begin{array}{l}\text { Proteína } \\
\text { Totais (g/dL) }\end{array}$ & $6,1 \pm 0,1$ & $5,68 \pm 0,12$ & $5,57 \pm 0,12$ & $7,23 \pm 0,11$ & $7,14 \pm 0,08$ & $7,22 \pm 0,14$ \\
\hline $\begin{array}{l}\text { Fosfatase } \\
\text { Alcalina (U/L) }\end{array}$ & $184,0 \pm 8,0$ & $177,56 \pm 14,12$ & $\begin{array}{c}175,98 \pm 9,2 \\
6\end{array}$ & $\begin{array}{c}257,36 \pm 12,81 \\
*\end{array}$ & $\begin{array}{c}231,95 \pm 17,46 \\
*\end{array}$ & $\begin{array}{c}205,29 \pm 12 \\
28\end{array}$ \\
\hline $\begin{array}{l}\text { ALT } \\
(\mathrm{U} / \mathrm{L})\end{array}$ & $61,0 \pm 2,4$ & $28,40 \pm 2,09$ & $30,83 \pm 1,38$ & $50,32 \pm 1,82$ & $60,24 \pm 3,24^{*}$ & $47,34 \pm 4,52$ \\
\hline $\begin{array}{l}\text { AST } \\
(\mathrm{U} / \mathrm{L})\end{array}$ & $152,4 \pm 6,6$ & $156,18 \pm 6,62$ & $\begin{array}{c}136,36 \pm 4,9 \\
2\end{array}$ & $160,02 \pm 10,99$ & $\begin{array}{c}188,93 \pm 10,85 \\
*\end{array}$ & $\begin{array}{c}168,52 \pm 5,5 \\
4\end{array}$ \\
\hline $\begin{array}{l}\text { Creatinina } \\
(\mathrm{mg} / \mathrm{dL})\end{array}$ & $0,3 \pm 0,1$ & $0,27 \pm 0,04$ & $0,24 \pm 0,04$ & $0,27 \pm 0,01$ & $0,22 \pm 0,02$ & $0,21 \pm 0,02$ \\
\hline $\begin{array}{l}\text { Uréia } \\
(\mathrm{mg} / \mathrm{dL})\end{array}$ & $45,2 \pm 2,3$ & $43,01 \pm 2,46$ & $40,83 \pm 1,50$ & $42,20 \pm 1,45$ & $38,36 \pm 2,56$ & $44,33 \pm 3,57$ \\
\hline $\begin{array}{l}\text { Ácido úrico } \\
(\mathrm{mg} / \mathrm{dL})\end{array}$ & $0,8 \pm 0,1$ & $0,53 \pm 0,04$ & $0,60 \pm 0,08$ & $0,90 \pm 0,07$ & $1,12 \pm 0,10$ & $1,04 \pm 0,05$ \\
\hline $\begin{array}{l}\text { GGT } \\
(\mathrm{U} / \mathrm{L})\end{array}$ & $20,0 \pm 0$ & $18,08 \pm 7,09$ & $11,13 \pm 4,10$ & $2,24 \pm 1,09$ & $0,82 \pm 0,75$ & $2,60 \pm 0,75$ \\
\hline $\begin{array}{l}\text { Albumina } \\
(\mathrm{g} / \mathrm{dL})\end{array}$ & $3,0 \pm 0$ & $2,83 \pm 0,03$ & $2,76 \pm 0,04$ & $2,80 \pm 0,04$ & $2,78 \pm 0,05$ & $2,83 \pm 0,09$ \\
\hline
\end{tabular}

Valores são expressos em média \pm erro padrão da média. Valores submetidos a ANOVA duas vias com pós-teste Boferroni significância*(p<0,05) grupos (FGBF 2,5\%, 5\%, $10 \%$ ou fitoterápico comercial x veículoda formulação.

\#(Branco, 2011).

Quanto às transaminases verificou-se que houve diferenças estatisticamente significativas para as variáveis ALT e AST no grupo da formulação $5 \%$ com aumento em relação aos valores referenciais apenas na AST. A ALT é considerada hepato-específica porque um significativo aumento em sua atividade sérica somente é observado na degeneração hepatocelular (Kaneko et al., 1997). O aumento nestas enzimas apresentados pelos animais tratados topicamente com o gel FGFB a 5\%, deve-se provavelmente a absorção do medicamento e posterior lesão dos hepatócitos, grave o bastante para provocar o aumento também da enzima AST. Valores elevados de AST e baixos valores de colesterol observados nesta pesquisa, revelam transtornos na função hepática (Bush, 1991). O ato de lambedura dos roedores pode ter contribuído na possível intoxicação dos animais.

A enzima AST é encontrada em maior quantidade nas mitocôndrias, cerca de $80 \%$, e não é liberada tão rápido como a ALT, que é uma enzima puramente citosólica. A AST está presente em altas concentrações em um grande número de tecidos, como coração, fígado, músculo esquelético, rins e pâncreas. A ALT é primariamente limitada ao citosol dos hepatócitos, é considerada um indicador altamente sensível de dano hepatocelular e, dentro de certos limites, pode fornecer uma taxa quantitativa do grau de danificação sofrido pelo fígado (Al-habori et al., 2002). Esses resultados bioquímicos diferem aos de Gomes et al. (2014) o qual observou um decréscimo de triglicerídeos, glicose, ALT e AST nos animais que receberam a dose de $5 \mathrm{~g} / \mathrm{kg}$ por via 
oral do extrato da casca da favela em roedores. A divergência dos resultados possivelmente se relaciona ao fato de terem sido empregadas diferentes metodologias.

Em relação ao estudo histopatológico do tecido hepático, não foram observadas alterações nos ratos tratados no controle positivo (Figura $5 \mathrm{~A}$ ), negativo (Figura $5 \mathrm{~B}$ ), extratos de FGBF 2,5\% (Figura 5 C) e 10\% (Figura 5 D). No entanto, nos animais do Grupo 5\% observou-se leve infiltrado inflamatório subcapsular, com hepatócitos com morfologia normal, com inclusões lipídicas, núcleos e nucléolos evidentes (Figura $5 \mathrm{E}$ ). De acordo com os achados descritos, sugere-se que os extratos $P$. neochilus e $C$. quercifolius não ocasionaram toxicidade às células hepáticas. Resultados similares obtidos por Gomes et al. (2014) utilizando o extrato bruto de folhas e cascas da favela por via oral, não detectou alterações do tecido hepático, confirmando que os extratos não ocasionaram toxicidade às células hepáticas.
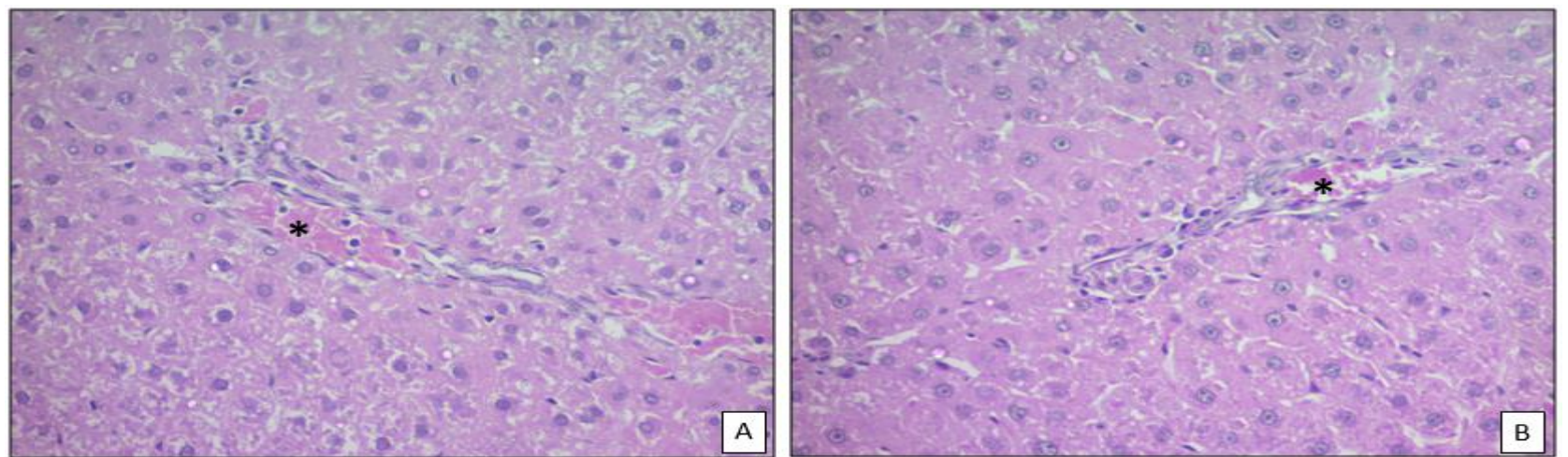

Figura 5. A e B. Fotomicrografias de fígado, apresentando hepatócitos morfologicamente normais próximos a vênula (asteriscos) de uma tríade portal. AU 400X; Coloração H.E.
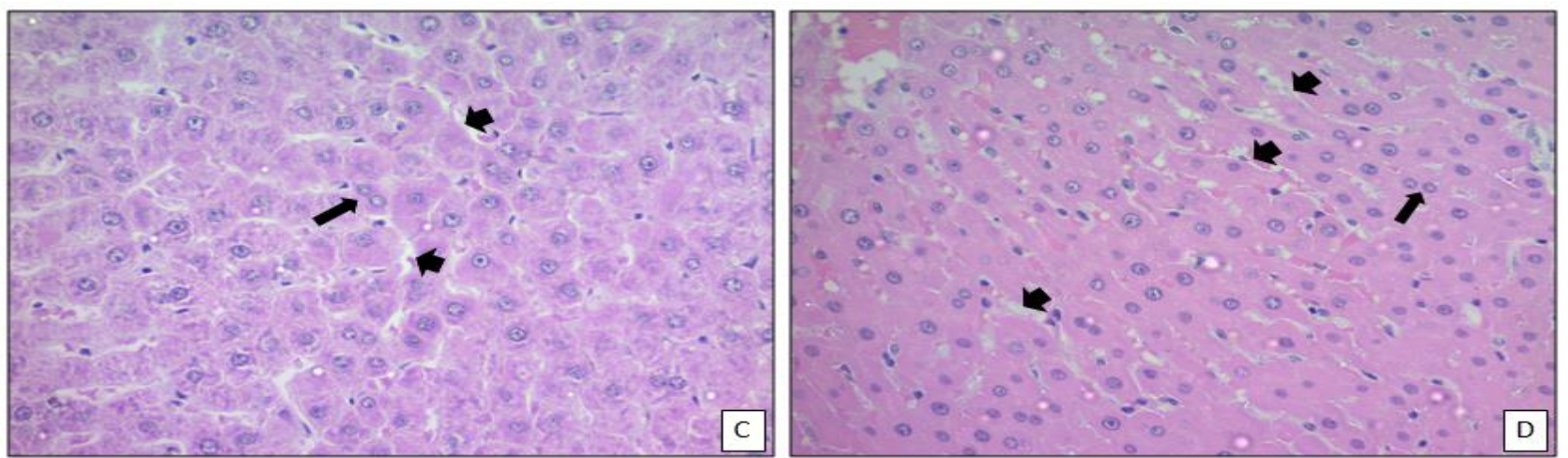

Figura 5. C e D. Fotomicrografias de fígado, apresentando cordões de hepatócitos com morfologias preservadas, hepatócitos binucleados (setas longas) e capilares sinusoides (setas curtas). AU 400X; Coloração H.E.
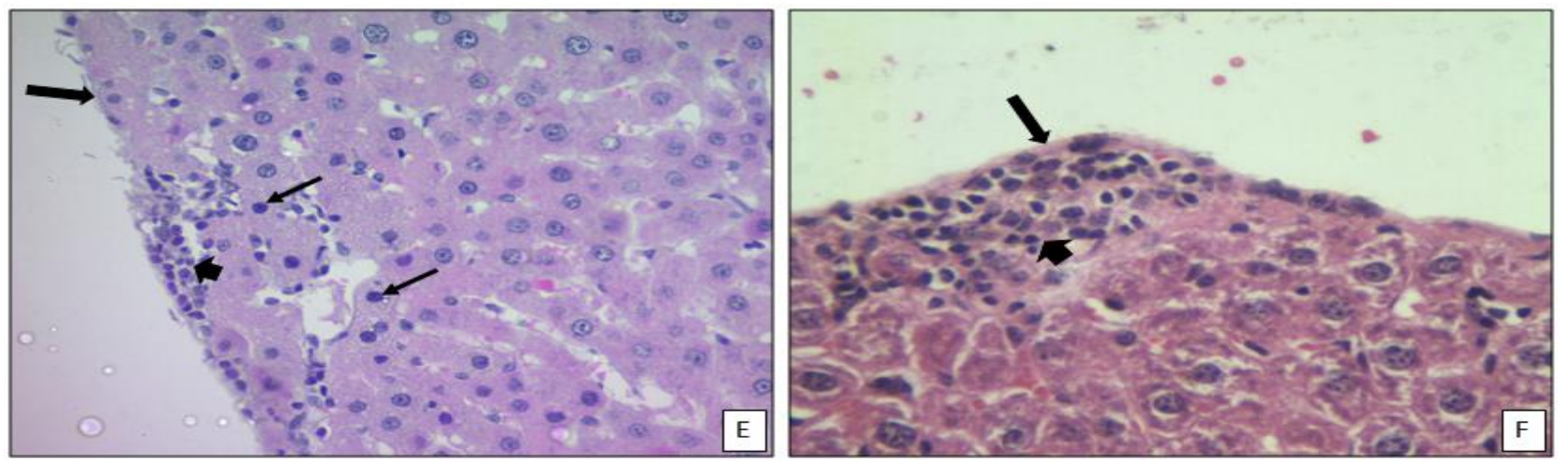

Figura 5. E e F. Fotomicrografias de fígado, apresentando infiltrado inflamatório mononuclerar (seta curta) abaixo da cápsula (seta longa); hepatócitos com picnose nuclear (setas finas). AU 400X e 1000X respectivamente; Coloração H.E. 
Dessa forma, o estudo da toxicidade aguda indica que o extrato etanólico bruto de $P$. neochilus e $C$. quercifolius não causaram toxicidade, porém, outros estudos com diferentes metodologias devem ser realizados, com o intuito de determinar uma melhor segurança dos extratos.

\section{Conclusão}

Os extratos hidroalcoólicos de $P$. neochilus e $C$. quercifolius causaram toxicidade aguda oral relativamente baixa em roedores. Apesar disso, em função das alterações orgânicas observadas faz-se necessário a realização de outros ensaios de toxicidade sub-crônica e crônica no intuito de determinar o perfil de segurança dos extratos para que os mesmos possam se tornar agentes seguros para uso terapêutico.

\section{Conflito de Interesse}

Os autores declaram não existir conflito de interesse.

\section{Comitê de Ética}

$\mathrm{O}$ projeto de pesquisa foi aprovado pela Comissão de Ética no Uso de Animais (CEUA) da Universidade Federal de Pernambuco (UFPE), Processo no 23076.011939/2014-25.

\section{Referências}

Agra, M.F.; Silva, K.N.; Ionaldo, J.L.D.B; Freitas, P.F.; Barbosa-filho, J.M. Survey of medicinal plants used in the region Northeast of Brazil. Revista Brasileira de Farmacognosia, 18: 472-508, 2008.

Al-habori, M.; Al-aghbari, A.; Al-mamary, M.; Baker, M. Toxicological evaluation of Catha edulis leaves: a long term feeding experiment in animals. Journal of ethnopharmacology, 83: 209-217, 2002.

Almeida, R.N. Psicofarmacologia: fundamentos práticos. Rio de Janeiro: Guanabara Koogan S. A, 2006. cap.11, p. 131-137.

Andrade, S.F. Manual de terapêutica veterinário. São Paulo: Roca, 2002.

Ascensão, L.; Figueiredo, A.C.; Barroso, J.G; Pedro, L.G.; Schripsema, J.; Deans, S.G.; Scheffer, J.J.C. Plectranthus madagascariensis: morphology of the glandular trichomes, essential oil composition, and its biological activity. Journal of Plant Sciences 159: 31-38, 1998.

Ascensão, L.; Mota, L.; Castro, M. Glandular trichomes on the leaves and $\mathrm{fl}$ owers of
Plectranthus ornatus: morphology, distribution and histochemistry. Annalls of Botany. 84: 437-447, 1999.

Bautista, A.R.P.L.; Miranda, M.S.; Batista, M.S.; Moreira, E.L.T.; Silva, I.M; Gomes, I.C.S. Avaliação da toxicidade oral subcrônica da bixina para ratos. Revista Brasileira de Ciências Farmacêuticas. 40: 229-233, 2004.

Brito, A.R.M.S. Toxicologia pré-clínica de plantas medicinais. In: Di stase LC (org.). Plantas medicinais: arte e ciência. São Paulo: UNESP, 1995. p.100-107.

Bush, B.M. Interpretation of laboratory results for small animal clinicians. Oxford: Blackwell Scientific, 1991. 215p.

Codd, L.E. Lamiaceae. In: Leistner OA (ed.). Flora of Southern Africa. Pretoria: Botanical Research Institute, Department of Agriculture and Water Supply, 28: 137-151, 1985.

Dipasquale, L.C.; Hayes, A.W. Acute toxicity and eye irritancy. In: Hayes, A.W. Principles and methods of toxicology. 4.ed. London: Taylor \& Francis, 2001. p. 853-916.

Duque, J.G. Perspectivas nordestinas. 2 ed. Fortaleza: Banco do Brasil, 2004. 423p.

Eming, S.A.; Krieg, T.; Davidson, J.M. Inflammation in wound repair: molecular and cellular mechanisms. Journal of Investigative Dermatology 127: 514-525, 2007.

Ferreira, A.L.; Rocha, C.P.; Vieira, L.M.; Dusse, L.M.S.A; Junqueira, D.R.G; Carvalho, M.G. Alterações hematológicas induzidas por medicamentos convencionais e alternativos. Revista Brasileira de Farmácia, 94: 94101, 2013.

Franco, E.S.; Aquino, C.M.F.; Medeiros, P.L.; Evêncio, L.B.; Góes, A.J.S.; Maia, M.B.S. Effect of a Semisolid Formulation of Linum usitatissimum L. (Linseed) Oil on the Reapair of Skin Wounds. Evidence-Based Complementary and Alternative Medicine. 12: 1-7, 2012.

Galiano, R.D.; Michaels, L.V.; Dobryansky, M.; Levine, J.P.; Gurtner, G.C. Quantitative and reproducible murine modelo $\mathrm{f}$ incisional wound healing. Wound repair and regeneration. 12: 485-492, 2004.

Gomes, L.M.A.; Andrade, T.M.D.; Silva, J.C.; Lima, J. T.; Quintans-Junior, L.J.; Almeida, J.R.G.S. 2014. A. Phytochemical screening 
and anti-inflammatory activity of Cnidoscolus quercifolius (Euphorbiaceae) in mice. Pharmacognosy Research. 6: 345349, 2014.

Jahn, A.I.; Gunzel, P.K.H. The value of spermatology in male reproductive toxicology: do spermatologic examination in fertility studies provide new and additional information relevante for safety assessment. Reproductive Toxicology. 11: p.171-178, 1997.

Kaneko, J.J.; Harvey, J.W.; Bruss, M.L. (Eds.) Clinical Biochemistry of Domestic Animals. San Diego: Academic Press, 1997. 932p.

Kaneda, K.; Serizawa, S.; Maruyasu, S.; Hasegawa, M.; Motono, M. Hair growth stimulating compositions containing (benzylamino) purines and Labiatae extracts. Japanase Kokai Tokkyo Koho, p. 18, 2005. Klaassen, C.D.; Watkins, I.E.J.B. Absorção, Distribuição e Excreção dos Tóxicos. In: Toxicologia: A ciência básica dos tóxicos de Casaret e Doull. $5^{\text {a }}$ ed. Cidade: McGrawHill, 2001. p. 79-100.

Lorenzi, H.; Matos, F.J.A. Plantas medicinais no Brasil: nativas e exóticas. São Paulo: Instituto Plantarum, 2002.

Martins, R.T.; Almeida, D.B.; Monteiro, F.M.R; Kowacs, P.A; Ramina, R. Receptores opioides até o contexto atual. Revista Dor, 13: 75-9, 2012.

Meyer, D.J.; Harvey, J.W. Veterinary laboratory medicine. 2. ed. Philadelphia: W.B. Manual de Patologia Clínica Veterinária, 1998. Saunders. p.372.

Nussey, G.; Van vuren, J.H.J.; Du preez, H.H. Effect of copper on the differential white blood cell counts of the Mozambique tilapia (Oreochromis mossambicus). Comparative
Biochemistry and Physiology. 111: 381388, 1995.

Okamoto, H.; Tananka, K.; Hasegawa, J. Chrysanthemun and other plant extracts as NF-kB activation inhibitors for treatment of inflammatory diseases and skin aging and related diseases. Jpn. Kokai Tokkyo Koho. 2005.

OECD (Organisation for economic cooperation and development). Guidelines for the Testing of Chemicals. OECD 423. Acute Oral ToxicityAcute Toxic Class Method. Organisation for Economic Cooperation and Development, Paris, 2001.

OECD (Organization for economic co-operation and development) 2001. Guideline for Testing of Chemicals: Acute Oral Toxicity-Acute Toxic Class Method. Guideline: 423. Disponível em: http://iccvam.niehs.nih.gov/SuppDocs/FedD ocs/OECD/OECD_GL423.pdf. Acesso em janeiro de 2017.

Rodrigues, E.; Duarte-almeida, J.M.; Pires, J.M. Perfil farmacológico e fitoquímico de plantas indicadas pelos caboclos do Parque Nacional do Jaú (AM) como potenciais analgésicas. Parte 1. Revista Brasileira de Farmacognosia, 20: 981-991, 2010.

Sangeetha, M.K.; Vallabi, D.E.; Sali, V.K.; Thanka, J.; Vasanthi, H.R. Sub-acute toxicity profile of a modified resveratrol supplement. Food and Chemical Toxicology, 59: 492-500, 2013.

Webster, G.L.; Rupert, E.A. Phyllogenetic significance of pollen nuclear number in the Euphorbiaceae. Evolution, 27: 524-531, 1973.

Werner, S.; Grose, R. Regulation of wound healing by growth factors and cytokines. Physiological Reviews, 83: 835-870, 2003. 